\section{P4.053 DRUG RESISTANCE AMONG WOMEN ATTENDING ANTENATAL CLINIC}

doi:10.1136/sextrans-2013-051184.0951

P Enyan. University of Ghana, Accra, Ghana

Background WHO HIV drug resistance (HIVDR) threshold survey suggests that transmission of drug-resistance strains is likely to be limited. However, as access to ART is expanded, increased emergence of HIVDR is feared as a potential consequence. We have performed a surveillance survey of transmitted HIVDR among recently infected persons in the geographic setting of Accra, Ghana.

Methods As part of a cross-sectional survey, 2 large voluntary counselling and testing centres in Accra enrolled 50 newly HIVdiagnosed, antiretroviral drug-naïve adults aged 18 to 25 years. Virus from plasma samples with $>1,000$ HIV RNA copies $/ \mathrm{mL}$ (Roche Amplicor v1.5) were sequenced in the pol gene. Transmitted drug resistance-associated mutations (TDRM) were identified according to the WHO 2009 Surveillance DRM list, using Stanford CPR tool (v 5.0 beta). Phylogenetic relationships of the newly characterised viruses were estimated by comparison with HIV-1

Results Subtypes were predominantly D (39/70, 55.7\%), A (29/70, $41.4 \%)$, and $\mathrm{C}(2 / 70 ; 2,9 \%)$. Seven nucleotide sequences harboured a major TDRM (3 NNRTI, 3 NRTI, and 1 PI- associated mutation); HIVDR point prevalence was $10.0 \%$ ( $95 \%$ CI $4.1 \%$ to $19.5 \%$ ). The identified TDRM were D67G (1.3\%), L210W (2.6\%); G190A (1.3\%); G190S (1.3\%); K101E (1.3\%), and N88D (1.3\%) for PI.

Conclusions In Accra the capital city of Ghana, we found a rate of transmitted HIVDR, which, according to the WHO threshold survey method, falls into the moderate (5 to $15 \%$ ) category. This is a considerable increase compared to the rate of $<5 \%$ estimated in the 2006-7 survey among women attending an antenatal clinic in mamobi. As ART programmes expand throughout Africa, incident infections should be monitored for the presence of transmitted drug resistance in order to guide ART policies.

\section{P4.054 HIV/AIDS EPIDEMIOLOGICAL CORRELATES OF SEXUAL BEHAVIOUR IN URBAN MIGRANTS TAMILNADU, INDIA}

doi:10.1136/sextrans-2013-051184.0952

R Athimulam Kulasekaran. Annamalai University, Chidambaram, India

Background Migrant population groups potentate the effect of ever-growing HIV/AIDS epidemic. Hotel migrant workers being one such group are at risk because of their age, economic independence, low level of education, group living and residence in a place away from their family.

Methods Study was conducted one of high HIV prevalence state of India during 2009 Criteria used in selecting study areas include number of hotels, volume of hotel migrant workers and prevalence rate of HIV. By simple random method, 609 respondents are selected from 20 PSUs who made more than two moves during last three years. Odds ratio with $95 \%$ confidence interval are computed to assess risk association.

Results Sexual activity was widespread among hotel migrant workers, irrespective of their marital status. Mean age at first sexual experience was 23.49 years. One-third had sexual contact with FSWs in past 12 months and fifty percent had sexual relationship with Non-FSWs. Unmarried migrants started their sexual actions with FSWs/Non-FSWs at less than 19 years. Condom usage was high with FSWs (68.3percent) than with Non-FSWs (20.0percent). It indicates migrants' perception of chance of getting HIV/AIDS varies according to their partners. Overall, 30percent of migrants suffered by any one sexual health/STIs related symptoms. Regression results shows that degree of mobility have an extreme positive relation with incidence of self-reported symptoms of STIs among clients of FSWs/Non-FSWs. The workers who have two moves are 4.5 times more likely to report symptoms of STIs. Exposure to pornography related matters is much strong predictor of STIs symptoms. Degree of mobility has extreme positive relation with incidence of STIs among clients of FSWs/Non-FSWs and risk of getting HIV will be more.

Conclusion It is recommended that HIV prevention interventions might benefit these populations if providing with the help of local para-medical professionals in their working place itself.

\section{P4.055 ASSOCIATION OF ALCOHOL USE AND UNSAFE ANAL SEX AMONG MEN HAVING SEX WITH MEN IN MANIPUR AND NAGALAND, NORTHEAST INDIA}

doi:10.1136/sextrans-2013-051184.0953

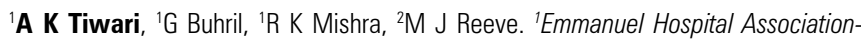
Project ORCHID, Guwahati, India; ${ }^{2}$ Nossal Institute for Global Health, University of Melbourne, Melbourne, Australia

Background In 2008, the HIV prevalence among men having sex with men (MSM) in Manipur state, India was $17.21 \%$. Project ORCHID is an Indian HIV prevention project funded by Avahan India, which supports local partner NGOs to deliver a range of harm reduction interventions targeting injecting drug users, men having sex with men and female sex workers. It reaches up to $1450 \mathrm{MSM}$ in two districts of Manipur and Nagaland. To measure HIV risk behaviour among this group, a census survey was carried out in 2010.

Methods Male sex workers $(n=1446)$ enrolled in the targeted intervention programme were interviewed through questionnaires. Peer educators and community volunteers met MSMs individually and administered the questionnaires. Variables were analysed using multivariate linear regression.

Results 1407 MSM returned valid responses regarding their sexual behaviour, out of whom $62.2 \%(n=899)$ reported unsafe anal sex in the previous week. Out of the 1446 interviewed $61 \%(n=858)$ reported alcohol consumption at the most recent sexual encounter with another MSM.

Variables including age, identity, literacy, marital status, number of partners, and alcohol consumption were tested for association with unsafe anal sex by bivariate analysis, and only number of partners, identity and consumption of alcohol at last sex were found significant and were included in the regression model. Age, although not significant, was also included.

Linear regression analysis $(n=1407)$ showed that identity, having more partners, and consumption of alcohol at the time of the most recent sex act were all significantly associated with higher rates of unsafe sex.

Conclusion HIV prevention programmes among MSM in India need to address issues of alcohol use and design appropriate interventions to reduce vulnerabilities of MSM in this regard. Interventions should also consider the particular risks and needs of those with sexual identities associated with more ano-receptive sex and high volume of partners.

\section{P4.056 THE UNIQUE HIV RISK PROFILE OF FEMALE INJECTING DRUG USERS IN MANIPUR, NORTHEAST INDIA}

doi:10.1136/sextrans-2013-051184.0954

${ }^{1}$ A K Tiwari, ${ }^{1 R} \mathrm{~K}$ Mishra, ${ }^{2} \mathrm{M} \mathrm{J}$ Reeve. ${ }^{1}$ Emmanuel Hospital Association- Project ORCHID, Guwahati, India; ${ }^{2}$ Nossal Institute for Global Health, University of Melbourne, Melbourne, Australia

Background Injecting drug users (IDUs) are at high HIV risk, but particularly women who inject drugs. In 2009, the HIV prevalence among IDUs in Churachandpur district, Manipur was 39.9\%, the highest in India. Project ORCHID and its partner SHALOM provide harm reduction services to over 700 IDUs in Churachandpur district, including 170 females. This study identifies differences between male and female IDUs registered with SHALOM. 
Methodology A total of 721 IDUs (551 male, 170 female) enrolled in SHALOM were interviewed by peer educators in early 2012 as part of programme risk assessments during routine outreach. Bivariate and multivariate analyses were conducted to compare the male and female clients.

Results Female IDUs engaged in much higher risk behaviour than male IDUs, injecting over twice the rate of men (20.7 vs. 10.0 times per week, $\mathrm{p}<0.001)$. Sexual activity was higher among women - a mean of 18 sex acts per week compared to less than one per week among men $(p<0.001)$. Women were significantly more likely to be poly-drug users and regularly drink alcohol. Women were significantly less educated, less likely to have a regular sexual partner and more likely to be widowed or divorced. They were more likely to be working full-time (mostly as sex workers).

Linear regression analysis for female IDUs $(n=169, R 2=0.46)$ showed that older age, more sex acts per week and poly-drug use were significantly associated with greater injecting frequency.

Linear regression analysis for male IDUs $(n=386, R 2=0.21$ ) showed that older age and heroin use were significantly associated with greater injecting frequency.

Conclusion Female IDUs have increased risk of HIV compared to their male counterparts due to more frequent injecting (associated with higher income from sex work), sex (mostly paid) and alcohol use. Such high risks require targeted interventions to meet the specific needs of female IDUs.

\section{Abstract P4.056 Table 1}

\begin{tabular}{lll}
\hline \multicolumn{2}{l}{ Linear regression analysis for female IDUs } \\
\hline No of Injections per week & Coefficients & P > t \\
\hline Age & 0.384 & 0.004 \\
No of sex acts per week & 0.487 & 0.000 \\
Type of drug use & 4.413 & 0.003 \\
\hline
\end{tabular}

\section{Abstract P4.056 Table 2}

\begin{tabular}{lll}
\hline \multicolumn{2}{l}{ Linear regression analysis for male IDUs } \\
\hline No of Injections per week & Coefficients & $\mathbf{P}>\mathbf{t}$ \\
\hline Age & 0.184 & 0.001 \\
Type of drug use & -5.632 & 0.000 \\
\hline
\end{tabular}

\section{P4.057 INVESTIGATING THE HIV KNOWLEDGE-PERSONAL RISK AWARENESS GAP AMONG BLACK AFRICANS IN LONDON, UK}

doi:10.1136/sextrans-2013-051184.0955

'P Tobi, ${ }^{2} \mathrm{~F}$ Phillips, ${ }^{2} \mathrm{C}$ Mead, ${ }^{2} \mathrm{~S}$ Lwembe, ${ }^{3 \mathrm{R}}$ Jones, ${ }^{4} \mathrm{~T}$ Ojwang, ${ }^{5} \mathrm{~J}$ Nyabwa. ${ }^{1}$ University of East London, London, UK; ${ }^{2}$ Inner North West London PCTS, London, UK; ${ }^{3}$ Chelsea and Westminster Hospital, London, UK; ${ }^{4}$ Opportunity for All, London, UK; ${ }^{5}$ West London African HIV Prevention Partnership, London, UK

Background Black Africans comprise $5.5 \%$ of the London population, but account for $1 / 3^{\text {rd }}$ of newly diagnosed HIV cases and $1 / 3^{\text {rd }}$ of diagnosed people accessing care. Current policy encourages targeted interventions to raise HIV awareness and promote uptake of testing in this group. 'Love Safely' is a 10-year West London community outreach partnership promoting HIV awareness and behaviour change. It deploys outreach workers working with a toolkit to engage people. Methods The study was conducted in 2012. A mixed-methods approach involved analysis of data on 1078 clients and interviews/ focus groups with 54 informants - partner organisations, outreach workers, clients, and referral services. Behaviour change was measured through three indicators: (a) recommending family members/friends for a similar visit, (b) exhibiting more careful sexual behaviour (consistent/correct condom use), and (c) accessing HIV testing. Process measures were explored to understand pathways of change.

Results The service was effective in penetrating some hard-toreach groups (men) but less so with others (older people, Muslim faith). Several aspects of knowledge, attitudes and behaviour were influenced by gender, length of UK residency and HIV prevalence in country of origin. Sexual healthcare was accessed from fixed settings but access through mobile units had increased. A high level of HIV knowledge and willingness to discuss HIV issues within social networks was demonstrated, but despite some evidence of behaviour change, awareness of personal risk was low. The main reasons for not attending HIV testing highlighted multi-level (individual, community and service) barriers and had remained unchanged compared to findings from a 2004 evaluation.

Conclusion Behaviour patterns are hard to shift and require persistent, robust action to bridge the knowledge-motivation-behaviour gap. Targeted intervention, while necessary, is insufficient. Emphasis on pluralistic, multi-level, joined up approaches may be more effective. Notwithstanding prevailing HIV stigma, people's social networks offer a potentially supportive role in prevention.

\section{P4.058 INCORRECT INFERENCES ABOUT MALE CIRCUMCISION AND FEMALE HIV INFECTION RISK: EVIDENCE FROM A RANDOMISED TRIAL IN MALAWI}

doi:10.1136/sextrans-2013-051184.0956

'B G Maughan-Brown, ${ }^{2} \mathrm{~A}$ Venkataramani, ${ }^{3} \mathrm{~S}$ Godlonton, ${ }^{3} \mathrm{R}$ Thornton. 'University of Cape Town, Cape Town, South Africa; ${ }^{2}$ Harvard University, Boston, MA, United States; ${ }^{3}$ University of Michigan, Ann Arbor, MI, United States

Background Incorrect inferences drawn from large-scale HIV prevention messages may hinder their efficacy. We examined whether individuals who are told that medical male circumcision (MMC) partially reduces female-to-male HIV transmission also erroneously infer a reduction in male-to-female HIV transmission risk.

Methods In 2008, nearly 1,000 men in rural Malawi were randomised to receive the information that MMC reduces female-tomale HIV transmission risk, with follow-up in 2009. Data was collected on perceived HIV transmission risks faced by men and women by the circumcision status of the male partner. Descriptive and multivariate variable regression analyses were used to assess whether beliefs about male circumcision and female HIV risk varied by receipt of information about MMC and by whether individuals believed that that MMC partially protects men from HIV infection.

Results Men randomised to information about MMC were more likely to believe that circumcised men faced reduced HIV transmission risks relative to uncircumcised men $(50 \%$ versus $65 \%, \mathrm{p}<0.01)$. They were also more likely to believe that sex with a circumcised male would confer lower transmission risks for women vis-à-vis sex with an uncircumcised male (38\% versus $50 \%$, p < 0.01$)$. Multivariate Ordinary Least Squares (OLS) regression analyses supported these findings and demonstrated that those who internalised beliefs about the risk reduction benefits accruing to men believed, on average, that circumcision conferred a 22 percentage point greater risk reduction $(p<0.01)$ in the probability of HIV transmission from men to women than men who did not believe that MMC protects men. Moreover, instrumental variable analyses indicated that the result from the OLS regression were an underestimation of the true effect.

Conclusions Our results point toward the need for male circumcision campaigns to make explicit that male circumcision does not directly protect women from HIV infection. 